\title{
Surface vacuum energy and stresses on a plate uniformly accelerated through the Fulling-Rindler vacuum
}

\author{
A. A. Saharian ${ }^{1 *}$ and M. R. Setare ${ }^{2 \dagger}$ \\ ${ }^{1}$ Department of Physics, Yerevan State University, Yerevan, Armenia \\ ${ }^{2}$ Institute for Theoretical Physics and Mathematics, Tehran, Iran
}

July 6, 2018

\begin{abstract}
The vacuum expectation value of the surface energy-momentum tensor is evaluated for a massless scalar field obeying mixed boundary condition on an infinite plate moving by uniform proper acceleration through the Fulling-Rindler vacuum. The generalized zeta function technique is used, in combination with the contour integral representation. The surface energies for separate regions on the left and on the right of the plate contain pole and finite contributions. Analytic expressions for both these contributions are derived. For a minimally coupled scalar the surface energy-momentum tensor induced by vacuum quantum effects corresponds to a source of the cosmological constant type located on the plate and with the cosmological constant determined by the proper acceleration of the plate.
\end{abstract}

PACS number(s): 03.70.+k, 11.10.Kk

\footnotetext{
*E-mail: saharyan@www.physdep.r.am

${ }^{\dagger}$ E-mail: rezakord@ipm.ir
} 


\section{Introduction}

The use of general coordinate transformations in quantum field theory leads to an infinite number of unitary inequivalent representations of the commutation relations. Different inequivalent representations will in general give rise to different pictures with different physical implications, in particular to different vacuum states. For instance, the vacuum state for a uniformly accelerated observer, the Fulling-Rindler vacuum $[1,2,3,4]$, turns out to be inequivalent to that for an inertial observer, the familiar Minkowski vacuum. Quantum field theory in accelerated systems contains many special features produced by a gravitational field. This fact allows one to avoid some of the difficulties entailed by renormalization in a curved spacetime. In particular, the near horizon geometry of most black holes is well approximated by the Rindler metric and a better understanding of physical effects in this background could serve as a handle to deal with more complicated geometries like Schwarzschild. The Rindler geometry shares most of the qualitative features of black holes and is simple enough to allow detailed analysis. Another motivation for the investigation of quantum effects in the Rindler space is related to the fact that this space is conformally related to the de Sitter (dS) space and to the Robertson-Walker space with negative spatial curvature. As a result the expectation values of the energy-momentum tensor for a conformally invariant field and for corresponding conformally transformed boundaries on dS and Robertson-Walker backgrounds can be derived from the corresponding Rindler counterpart by the standard transformation (see, for instance, [5]).

The problem of vacuum polarization by an infinite plane boundary moving with uniform acceleration through the Fulling-Rindler vacuum was investigated by Candelas and Deutsch [6] for the conformally coupled $4 D$ Dirichlet and Neumann massless scalar and electromagnetic fields. In this paper only the region of the right Rindler wedge to the right of the barrier is considered. In Ref. [7] we have investigated the Wightman function and the vacuum expectation values of the energy momentum-tensor for the massive scalar field with general curvature coupling parameter, satisfying the Robin boundary conditions on the infinite plane in an arbitrary number of spacetime dimensions and for the electromagnetic field. Unlike Ref. [6] we have considered both regions, including the one between the barrier and Rindler horizon. The vacuum expectation values of the energy-momentum tensors for Dirichlet or Neumann scalar and electromagnetic fields for the geometry of two parallel plates moving by uniform acceleration are investigated in Ref. [8]. In particular, the vacuum forces acting on the boundaries are evaluated. They are presented as a sum of the interaction and self-action parts. The interaction forces between the plates are always attractive for both scalar and electromagnetic cases. In Refs. $[7,8]$ the mode summation method is used in combination with the generalized Abel-Plana summation formula [9]. This allowed us to present the vacuum expectation values in terms of the purely Rindler and boundary-induced parts. Due to the well-known non-integrable surface divergences in the boundary parts, the total Casimir energy cannot be obtained by direct integration of the vacuum energy density and needs an additional regularization. In Ref. [10] the Casimir energy is evaluated for massless scalar fields under Dirichlet or Neumann boundary conditions, and for the electromagnetic field with perfect conductor boundary conditions on one and two infinite parallel plates moving by uniform proper acceleration through the Fulling-Rindler vacuum in an arbitrary number of spacetime dimension. In Ref. [11] the conformal relation between dS and Rindler spacetimes and the results from Ref. [7] are used to generate the vacuum expectation values of the energy-momentum tensor for a conformally coupled scalar field in dS spacetime in presence of a curved brane on which the field obeys the Robin boundary condition with coordinate dependent coefficients.

For scalar fields with general curvature coupling in Ref. [12] it has been shown that in the discussion of the relation between the mode sum energy, evaluated as the sum of the zero-point energies for each normal mode of frequency, and the volume integral of the renormalized energy 
density for the Robin parallel plates geometry it is necessary to include in the energy a surface term concentrated on the boundary (see also the discussion in Ref. [13]). Similar issues for the spherical and cylindrical boundary geometries are discussed in Refs. [14, 15]. An expression of the surface energy-momentum tensor for a scalar field with general curvature coupling parameter in the general case of bulk and boundary geometries is derived in Ref. [16]. In the present paper, by using this expression and the zeta function technique, we evaluate the vacuum expectation value of the surface energy-momentum tensor for a plate moving by uniform proper acceleration through the Fulling-Rindler vacuum.

The paper is organized as follows. In Section 2 the surface energy density and vacuum stresses are evaluated for the region on the right of the plate ( $R R$ region). We construct an integral representation for the related zeta function and analytically continue it to the physical region. Similar problem for the region on the left of the plate (RL region) is investigated in Section 3. Section 4 concludes the main results of the paper.

\section{Surface energy-momentum tensor in the $\mathrm{RR}$ region}

Consider a real massless scalar field $\varphi(x)$ with curvature coupling parameter $\zeta$ satisfying the field equation

$$
\nabla_{l} \nabla^{l} \varphi+\zeta R \varphi=0
$$

with $R$ being the scalar curvature for a $(D+1)$-dimensional background spacetime, $\nabla_{l}$ is the covariant derivative operator associated with the corresponding metric tensor $g_{i k}$. For minimally and conformally coupled scalars one has $\zeta=0$ and $\zeta=(D-1) / 4 D$, respectively. Our main interest in this paper will be the surface Casimir energy and stresses induced on a single plate moving with uniform proper acceleration when the quantum field is prepared in the FullingRindler vacuum. We will assume that the field satisfies the mixed boundary condition

$$
\left(A_{s}+n^{l} \nabla_{l}\right) \varphi(x)=0
$$

on the plate, where $A_{s}$ is a constant, $n^{l}$ is the unit inward normal to the plate. For this boundary condition the vacuum expectation values of the bulk energy-momentum tensor in the both RR and RL regions are evaluated in Ref. [7]. In Ref. [16] it was argued that the energy-momentum tensor for a scalar field on manifolds with boundaries in addition to the bulk part contains a contribution located on the boundary. For the boundary $\partial M_{s}$ the surface part of the energymomentum tensor is presented in the form [16]

$$
T_{i k}^{(\mathrm{surf})}=\delta\left(x ; \partial M_{s}\right) \tau_{i k}
$$

with

$$
\tau_{i k}=\zeta \varphi^{2} K_{i k}-(2 \zeta-1 / 2) h_{i k} \varphi n^{l} \nabla_{l} \varphi
$$

and the "one-sided" delta-function $\delta\left(x ; \partial M_{s}\right)$ locates this tensor on $\partial M_{s}$. In Eq. (4), $K_{i k}$ is the extrinsic curvature tensor of the boundary $\partial M_{s}$ and $h_{i k}$ is the corresponding induced metric. Let $\left\{\varphi_{\alpha}(x), \varphi_{\alpha}^{*}(x)\right\}$ be a complete set of positive and negative frequency solutions to the field equation (1), obeying boundary condition (2). Here $\alpha$ denotes a set of quantum numbers specifying the solution. By expanding the field operator over the eigenfunctions $\varphi_{\alpha}(x)$, using the standard commutation rules and the definition of the vacuum state, for the vacuum expectation value of the surface energy-momentum tensor one finds

$$
\left\langle 0\left|T_{i k}^{(\text {surf })}\right| 0\right\rangle=\delta\left(x ; \partial M_{s}\right)\left\langle 0\left|\tau_{i k}\right| 0\right\rangle, \quad\left\langle 0\left|\tau_{i k}\right| 0\right\rangle=\sum_{\alpha} \tau_{i k}\left\{\varphi_{\alpha}(x), \varphi_{\alpha}^{*}(x)\right\},
$$


where $|0\rangle$ is the amplitude for the corresponding vacuum state, and the bilinear form $\tau_{i k}\{\varphi, \psi\}$ on the right of the second formula is determined by the classical energy-momentum tensor (4).

In the accelerated frame it is convenient to introduce Rindler coordinates $(\tau, \xi, \mathbf{x})$ which are related to the Minkowski ones, $\left(t, x^{1}, \mathbf{x}\right)$ by transformations

$$
t=\xi \sinh \tau, \quad x^{1}=\xi \cosh \tau,
$$

where $\mathbf{x}=\left(x^{2}, \ldots, x^{D}\right)$ denotes the set of coordinates parallel to the plate. In these coordinates one has $g_{00}=\xi^{2}, g_{i k}=-\delta_{i k}, i \neq 0$, and a wordline defined by $\xi, \mathbf{x}=$ const describes an observer with constant proper acceleration $\xi^{-1}$. Rindler time coordinate $\tau$ is proportional to the proper time along a family of uniformly accelerated trajectories which fill the Rindler wedge, with the proportionality constant equal to the acceleration. For the geometry under consideration the metric and boundary conditions are static and translational invariant in the hyperplane parallel to the plate. It follows from here that the corresponding part of the eigenfunctions can be taken in the standard plane wave form:

$$
\varphi_{\alpha}=C_{D} \phi(\xi) \exp [i(\mathbf{k x}-\omega \tau)], \quad \alpha=(\mathbf{k}, \omega), \quad \mathbf{k}=\left(k_{2}, \ldots, k_{D}\right) .
$$

The equation for $\phi(\xi)$ is obtained from field equation (1). The corresponding linearly independent solutions are the Bessel modified functions $I_{i \omega}(k \xi)$ and $K_{i \omega}(k \xi)$ of the imaginary order, where $k=|\mathbf{k}|$. The eigenfrequencies are determined from the boundary condition imposed on the field on the bounding surface. As such a surface we take a plane boundary with coordinate $\xi=a>0$ corresponding to a plate uniformly accelerated normal to itself with the proper acceleration $a^{-1}$. The plate divides the right Rindler wedge into two regions with $\xi>a(\mathrm{RR}$ region) and $0<\xi<a$ (RL region). The vacuum properties in these regions are different and we consider them separately.

For the RR region the unit normal to the boundary and nonzero components of the extrinsic curvature tensor have the form

$$
n^{l}=\delta_{1}^{l}, \quad K_{00}=\xi,
$$

and $\phi(\xi)=K_{i \omega}(k \xi)$. For a given $k a$, the corresponding eigenfrequencies $\omega=\omega_{n}=\omega_{n}(k a)$, $n=1,2, \ldots$, are determined from boundary condition (2) and are solutions to the equation

$$
A K_{i \omega}(x)+x K_{i \omega}^{\prime}(x)=0, \quad x=k a, \quad A=A_{s} a,
$$

where the prime denotes the differentiation with respect to the argument of the function. For $A_{s}>0$ this equation has purely imaginary solutions with respect to $\omega$. To avoid the vacuum instability, below we will assume that $A_{s} \leq 0$. Under this condition all solutions to (9) are real. The coefficient $C_{D}$ in Eq. (7) is determined by the normalization condition and is equal to [7]

$$
C_{D}^{2}=\frac{1}{(2 \pi)^{D-1}} \frac{\bar{I}_{i \omega_{n}}(k a)}{\left.\frac{\partial}{\partial \omega} \bar{K}_{i \omega}(k a)\right|_{\omega=\omega_{n}}},
$$

where for a given function $F(x)$ we use the notation

$$
\bar{F}(x)=A F(x)+x F^{\prime}(x) .
$$

Substituting the eigenfunctions into the mode-sum formula (5) and using the relation

$$
K_{i \omega_{n}}(k a) \bar{I}_{i \omega_{n}}(k a)=1,
$$

the vacuum expectation value of the surface energy-momentum tensor can be presented in the form

$$
\left\langle 0\left|\tau_{l}^{k}\right| 0\right\rangle=\frac{B_{D} I_{\mathrm{R}}(A)}{a^{D}}\left[2 \zeta \delta_{l}^{0} \delta_{0}^{k}+(4 \zeta-1) A \delta_{l}^{k}\right], \quad l, k=0,2, \ldots, D,
$$


with

$$
I_{\mathrm{R}}(A)=\int_{0}^{\infty} d x x^{D-2} \sum_{n=1}^{\infty} \frac{K_{i \omega_{n}}(x)}{\left.\frac{\partial}{\partial \omega} \bar{K}_{i \omega}(x)\right|_{\omega=\omega_{n}}},
$$

and $\left\langle 0\left|\tau_{1}^{1}\right| 0\right\rangle=0$. Here and below the quantities for the RR and RL regions are denoted by the indices $\mathrm{R}$ and $\mathrm{L}$, respectively, and we use the notation

$$
B_{D}=\frac{1}{(4 \pi)^{\frac{D-1}{2}} \Gamma\left(\frac{D-1}{2}\right)} .
$$

The surface energy-momentum tensor (13) has a diagonal structure:

$$
\left\langle 0\left|\tau_{l}^{k}\right| 0\right\rangle=\operatorname{diag}(\varepsilon, 0,-p, \ldots,-p),
$$

with the surface energy density $\varepsilon$ and stress $p$, and the equation of state

$$
\varepsilon=-\left[1+\frac{2 \zeta}{A(4 \zeta-1)}\right] p
$$

For a minimally coupled scalar field, this corresponds to a cosmological constant induced on the plate. In accordance with (13) for the vacuum stress one has

$$
p=\frac{B_{D}}{a^{D}} A(1-4 \zeta) I_{R}(A)
$$

The quantity (14) and, hence, the surface energy-momentum tensor diverges and needs some regularization. Many regularization techniques are available nowadays and, depending on the specific physical problem under consideration, one of them may be more suitable than the others. In particular, the generalized zeta function method $[17,18,19]$ is in general very powerful to give physical meaning to the divergent quantities. There are several examples of the application of this method to the evaluation of the Casimir effect (see, for instance, $[18,20,21,22,23,24,25,26,27,28])$. Here we will use the method which is an analog of the generalized zeta function approach. We define the function

$$
F_{\mathrm{R}}(s)=\int_{0}^{\infty} d x x^{D-2} \zeta_{\mathrm{R}}(s, x)
$$

where

$$
\zeta_{\mathrm{R}}(s, x)=\sum_{n=1}^{\infty} \frac{\omega_{n}^{-s} K_{i \omega_{n}}(x)}{\left.\frac{\partial}{\partial \omega} \bar{K}_{i \omega}(x)\right|_{\omega=\omega_{n}}} .
$$

The computation of vacuum expectation value for the surface energy-momentum tensor requires an analytical continuation of the function $F_{\mathrm{R}}(s)$ to the value $s=0$,

$$
I_{\mathrm{R}}(A)=\left.F_{\mathrm{R}}(s)\right|_{s=0} .
$$

The starting point of our consideration is the representation of the function (20) in terms of contour integral

$$
\zeta_{\mathrm{R}}(s, x)=\frac{1}{2 \pi i} \int_{C} d z z^{-s} \frac{K_{i z}(x)}{\bar{K}_{i z}(x)}
$$

where $C$ is a closed counterclockwise contour in the complex $z$ plane enclosing all zeros $\omega_{n}(x)$. The location of these zeros enables one to deform the contour $C$ into a segment of the imaginary axis $(-i R, i R)$ and a semicircle of radius $R$ in the right half-plane. We will also assume that the origin is avoided by the semicircle $C_{\rho}$ with small radius $\rho$. For sufficiently large $s$ the integral 
over the large semicircle in (22) tends to zero in the limit $R \rightarrow \infty$, and the expression on the right can be transformed to

$$
\zeta_{\mathrm{R}}(s, x)=\frac{1}{2 \pi i} \int_{C_{\rho}} d z z^{-s} \frac{K_{i z}(x)}{\bar{K}_{i z}(x)}-\frac{1}{\pi} \cos \frac{\pi s}{2} \int_{\rho}^{\infty} d z z^{-s} \frac{K_{z}(x)}{\bar{K}_{z}(x)} .
$$

Below we will consider the limit $\rho \rightarrow 0$. In this limit the first integral vanishes in the case $s=0$, and in the following we will concentrate on the contribution of the second integral. For the analytic continuation of this integral we employ the uniform asymptotic expansion of the MacDonald function for large values of the order [29]. We will rewrite this expansion in the form

$$
K_{z}(x) \sim \sqrt{\frac{\pi}{2}} \frac{e^{-z \eta(x / z)}}{\left(x^{2}+z^{2}\right)^{1 / 4}} \sum_{l=0}^{\infty} \frac{(-1)^{l} \widetilde{u}_{l}(t)}{\left(x^{2}+z^{2}\right)^{l / 2}},
$$

where

$$
t=\frac{z}{\sqrt{x^{2}+z^{2}}}, \quad \eta(x)=\sqrt{1+x^{2}}+\ln \frac{x}{1+\sqrt{1+x^{2}}}, \quad \tilde{u}_{l}(t)=\frac{u_{l}(t)}{t^{l}},
$$

and the expressions for the functions $u_{l}(t)$ are given in [29]. From these expressions it follows that the coefficients $\tilde{u}_{l}(t)$ have the structure

$$
\tilde{u}_{l}(t)=\sum_{m=0}^{l} u_{l m} t^{2 m}
$$

with numerical coefficients $u_{l m}$. From Eq. (24) and the corresponding expansion for the derivative of the MacDonald function we obtain the asymptotic expansion

$$
\bar{K}_{z}(x) \sim-\sqrt{\frac{\pi}{2}}\left(x^{2}+z^{2}\right)^{1 / 4} e^{-z \eta(x / z)} \sum_{l=0}^{\infty} \frac{(-1)^{l} \tilde{v}_{l}(t)}{\left(x^{2}+z^{2}\right)^{l / 2}},
$$

where

$$
\tilde{v}_{l}(t)=\frac{v_{l}(t)}{t^{l}}+A \tilde{u}_{l-1}
$$

and the expressions for $v_{l}(t)=t^{l} \sum_{m=0}^{l} v_{l m} t^{2 m}$ are presented in [29]. The recurrence formulae for the numerical coefficients $u_{l m}$ and $v_{l m}$ can be found in Ref. [10]. Note that the functions (28) have the structure

$$
\tilde{v}_{l}(t)=\sum_{m=0}^{l} \tilde{v}_{l m} t^{2 m}, \quad \tilde{v}_{l m}=v_{l m}+A u_{l-1, m} .
$$

From Eqs. (24) and (27) we can find the asymptotic expansion for the ratio in the second integral on the right of formula (23). For the further convenience we will write this expansion in the form

$$
\frac{K_{z}(x)}{\bar{K}_{z}(x)} \sim-\frac{1}{\left(x^{2}+z^{2}\right)^{1 / 2}} \sum_{l=0}^{\infty} \frac{(-1)^{l} U_{l}(t)}{\left(1+x^{2}+z^{2}\right)^{l / 2}},
$$

where the coefficients $U_{l}(t)$ are defined by the relation

$$
\sum_{l=0}^{\infty}(-1)^{l} \frac{\tilde{u}_{l}(t)}{r^{l}}\left[\sum_{l=0}^{\infty}(-1)^{l} \frac{\tilde{v}_{l}(t)}{r^{l}}\right]^{-1}=\sum_{l=0}^{\infty} \frac{(-1)^{l} U_{l}(t)}{\left(1+r^{2}\right)^{l / 2}}
$$


and similar to (26), (29), are polynomials in $t$ :

$$
U_{l}(t)=\sum_{m=0}^{l} U_{l m} t^{2 m} .
$$

The first three coefficients are given by expressions

$$
\begin{aligned}
U_{1}(t) & =\frac{1}{2}-A-\frac{t^{2}}{2} \\
U_{2}(t) & =\frac{3}{8}-A+A^{2}+\left(-\frac{5}{4}+A\right) t^{2}+\frac{7 t^{4}}{8} \\
U_{3}(t) & =\frac{5}{8}-\frac{3 A}{2}+\frac{3 A^{2}}{2}-A^{3}+\left(-\frac{25}{8}+3 A-\frac{3 A^{2}}{2}\right) t^{2}+\left(\frac{41}{8}-2 A\right) t^{4}-\frac{21 t^{6}}{8} .
\end{aligned}
$$

Now let us consider the function

$$
F_{\mathrm{R}}(s)=-\frac{1}{\pi} \cos \frac{\pi s}{2} \int_{0}^{\infty} d x x^{D-2} \int_{\rho}^{\infty} d z z^{-s} \frac{K_{z}(x)}{\bar{K}_{z}(x)}
$$

We subtract and add to the integrand in this equation the corresponding asymptotic expression. This allows us to split (34) into the following pieces

$$
F_{\mathrm{R}}(s)=F_{\mathrm{R}}^{(a s)}(s)+F_{\mathrm{R}}^{(1)}(s),
$$

where

$$
\begin{aligned}
& F_{\mathrm{R}}^{(a s)}(s)=\frac{1}{\pi} \cos \frac{\pi s}{2} \int_{0}^{\infty} d x x^{D-2} \int_{\rho}^{\infty} d z z^{-s} \frac{1}{r} \sum_{l=0}^{N} \frac{(-1)^{l} U_{l}(\cos \theta)}{\left(1+r^{2}\right)^{l / 2}} \\
& F_{\mathrm{R}}^{(1)}(s)=-\frac{1}{\pi} \cos \frac{\pi s}{2} \int_{0}^{\infty} d x x^{D-2} \int_{\rho}^{\infty} d z z^{-s}\left[\frac{K_{z}(x)}{\bar{K}_{z}(x)}+\frac{1}{r} \sum_{l=0}^{N} \frac{(-1)^{l} U_{l}(\cos \theta)}{\left(1+r^{2}\right)^{l / 2}}\right]
\end{aligned}
$$

and

$$
r=\left(x^{2}+z^{2}\right)^{1 / 2}, \quad \cos \theta=z / r .
$$

For $N \geq D-1$ the expression for $F_{\mathrm{R}}^{(1)}(s)$ is finite at $s=0$ and, hence, for our aim it is sufficient to subtract $N=D-1$ asymptotic terms. At $s=0$ the function $F_{\mathrm{R}}^{(1)}(s)$ is finite for $\rho=0$ and we can directly put this value. The integral over $z$ in the expression for $F_{\mathrm{R}}^{(a s)}(s)$ is finite in the limit $\rho \rightarrow 0$ for $0<\operatorname{Re} s<1$. For these values we can put $\rho=0$ in Eq. (36). After the integration over $z$, the contribution of the $l=0$ term in this formula can be presented in the form

$$
\frac{1}{2 \pi} B\left(\frac{1-s}{2}, \frac{s}{2}\right) \cos \frac{\pi s}{2} \int_{0}^{\infty} d x x^{D-2-s},
$$

with the beta function $B(x, y)$. Now using the standard dimensional regularization result that the renormalized value of the integrals of the type $\int_{0}^{\infty} d x x^{\beta}$ is equal to zero (see, e.g., [30]), we conclude that the contribution of the term with $l=0$ into Eq. (36) vanishes. This can be seen by another way, considering the case of a scalar field with nonzero mass $m$ and taking the limit $m \rightarrow 0$ after the evaluation of the corresponding integrals (for this trick in the calculations of the Casimir energy see, for instance, Refs. [31, 32]). For the massive case, in Eq. (7) one has $\phi(\xi)=K_{i \omega}\left(\xi \sqrt{k^{2}+m^{2}}\right)$ and the corresponding formulae are obtained from those given above in this section by replacement $x \rightarrow \sqrt{x^{2}+m^{2} a^{2}}$. With this replacement the integral corresponding to the contribution of $l=0$ term into Eq. (36) can be easily evaluated in terms of the gamma 
function and vanishes in the limit $m \rightarrow 0$ for $\operatorname{Re} s<D-1$. After performing to the polar coordinates $(r, \theta)$ and using relation (32), from (36) one finds

$$
F_{\mathrm{R}}^{(a s)}(s)=\frac{1}{\pi} \cos \frac{\pi s}{2} \sum_{l=1}^{N}(-1)^{l} \sum_{m=0}^{l} U_{l m} \int_{0}^{\infty} d r \frac{r^{D-s-2}}{\left(1+r^{2}\right)^{l / 2}} \int_{0}^{\pi / 2} d \theta \sin ^{D-2} \theta \cos ^{2 m-s} \theta .
$$

Evaluating the integrals by using the standard formulae, we find the expression

$$
F_{\mathrm{R}}^{(a s)}(s)=\frac{1}{\pi} \cos \frac{\pi s}{2} \sum_{l=1}^{N}(-1)^{l} B\left(\frac{D-s-1}{2}, \frac{l+s-D+1}{2}\right) \sum_{m=0}^{l} U_{l m} B\left(m-\frac{s-1}{2}, \frac{D-1}{2}\right),
$$

where the pole contribution is given explicitly in terms of the beta function. In the sum over $l$, the terms with odd $D-l \geq 1$ have simple poles at $s=0$ coming from the first beta function. Introducing a new summation variable $j=(D-l-1) / 2$, the corresponding residue can be easily found by using the standard formula for the gamma function:

$$
F_{\mathrm{R},-1}^{(a s)}=-\frac{2}{\pi}(-1)^{D} \Gamma\left(\frac{D-1}{2}\right) \sum_{j=0}^{j_{D}} \frac{(-1)^{j}}{\Gamma(j+1) \Gamma\left(\frac{D-1}{2}-j\right)} \sum_{m=0}^{D-2 j-1} U_{D-2 j-1, m} B\left(m+\frac{1}{2}, \frac{D-1}{2}\right),
$$

where

$$
j_{D}=\left\{\begin{array}{ll}
\frac{D-2}{2}, & \text { for even } D \\
\frac{D-3}{2}, & \text { for odd } D
\end{array} .\right.
$$

Laurent-expanding near $s=0$ we can write

$$
F_{\mathrm{R}}^{(a s)}(s)=\frac{F_{\mathrm{R},-1}^{(a s)}}{s}+F_{\mathrm{R}, 0}^{(a s)}+\mathcal{O}(s)
$$

with

$$
\begin{aligned}
F_{\mathrm{R}, 0}^{(a s)} & =-\frac{(-1)^{D}}{\pi} \Gamma\left(\frac{D-1}{2}\right) \sum_{j=0}^{j_{D}} \frac{(-1)^{j}}{\Gamma(j+1) \Gamma\left(\frac{D-1}{2}-j\right)} \sum_{m=0}^{D-2 j-1} U_{D-2 j-1, m} B\left(m+\frac{1}{2}, \frac{D-1}{2}\right) \\
& \times\left[\psi\left(m+\frac{D}{2}\right)+\psi(j+1)-\psi\left(m+\frac{1}{2}\right)-\psi\left(\frac{D-1}{2}\right)\right] \\
& +\frac{1}{\pi}\left(\sum_{l=1, D-l=\text { even }}^{D-1}+\sum_{l=D}^{N}\right)(-1)^{l} B\left(\frac{l-D+1}{2}, \frac{D-1}{2}\right) \sum_{m=0}^{l} U_{l m} B\left(m+\frac{1}{2}, \frac{D-1}{2}\right),
\end{aligned}
$$

where $\psi(x)=d \ln \Gamma(x) / d x$ is the diagamma function and the second sum in the braces of the third line is present only for $N \geq D$. The first term on the right of Eq. (45) with diagamma functions comes from the finite part of the Laurent expansion of the summands with odd $D-l$ in Eq. (41). Gathering all contributions together, near $s=0$ we find

$$
F_{\mathrm{R}}(s)=\frac{F_{\mathrm{R},-1}^{(a s)}}{s}+F_{\mathrm{R}, 0}^{(a s)}+F_{\mathrm{R}}^{(1)}(0)+\mathcal{O}(s) .
$$

Using this result, for the vacuum stress induced on the surface of a single plate one obtains

$$
p=p_{p}^{(\mathrm{R})}+p_{f}^{(\mathrm{R})},
$$


where for the pole and finite contributions one has

$$
\begin{aligned}
p_{p}^{(\mathrm{R})} & =\frac{B_{D}}{s a^{D}} A(1-4 \zeta) F_{\mathrm{R},-1}^{(a s)}, \quad A=a A_{s}, \\
p_{f}^{(\mathrm{R})} & =\frac{B_{D}}{a^{D}} A(1-4 \zeta)\left[F_{\mathrm{R}, 0}^{(a s)}+F_{\mathrm{R}}^{(1)}(0)\right],
\end{aligned}
$$

and the coefficients are defined by expressions (37), (42), (45). The corresponding formulae for the pole and finite parts of the surface energy density are obtained by using the equation of state (17). In particular, in the case of the Neumann boundary condition $(A=0)$ for the finite parts one has

$$
\varepsilon_{\mathrm{N} f}^{(\mathrm{R})}=\frac{2 \zeta B_{D}}{a^{D}}\left[F_{\mathrm{R}, 0}^{(a s)}+F_{\mathrm{R}}^{(1)}(0)\right]_{A=0}, \quad p_{\mathrm{N} f}^{(\mathrm{R})}=0 .
$$

In Fig. 1 we have plotted the dependence of the finite part of the quantity $a^{D} p_{f}^{(\mathrm{R})} /[A(1-4 \zeta)]$ on the parameter $-a A_{s}$ in the spatial dimension $D=3$. This quantity does not depend on the curvature coupling parameter and is positive for the RR region. For a minimally coupled scalar and $A>0$ this corresponds to the generation of the negative cosmological constant on the plate. The corresponding numerical value is determined by the proper acceleration of the plate. The surface energy per unit surface of the plate can be found integrating the energy density from Eq. (5),

$$
E^{(\mathrm{R}, \mathrm{surf})}=\int d^{D} x \sqrt{\left|\operatorname{det} g_{i k}\right|}\left\langle 0\left|T_{0}^{(\text {surf }) 0}\right| 0\right\rangle=a\left\langle 0\left|\tau_{0}^{0}\right| 0\right\rangle=a \varepsilon .
$$

In the case of the Neumann boundary condition, for the finite part of the surface energy in $D=3$ one obtains

$$
E_{\mathrm{N} f}^{(\mathrm{R}, \text { surf })}=\frac{0.111 \zeta}{a^{2}}
$$

The finite part of the corresponding total vacuum energy per unit surface is evaluated in Ref. [10] and is equal to $E_{\mathrm{N} f}^{(\mathrm{R})}=0.00213 / a^{2}$. This quantity is the sum of the volume and surface energies.

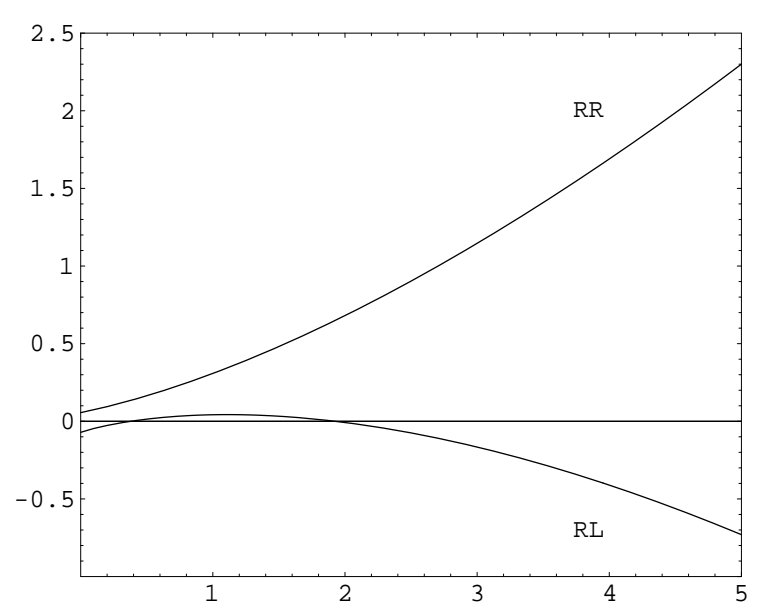

Figure 1: Finite parts of the surface vacuum stresses $a^{D} p_{f}^{(\mathrm{R}, \mathrm{L})} /(A(1-4 \zeta))$ for the RR and RL regions as functions on $-a A_{s}$. 


\section{Surface energy density and stresses in the RL region}

Consider the scalar vacuum in the region between the plate and the Rindler horizon corresponding to $\xi=0$ (RL region). As in the previous section we will assume that the field obeys boundary condition (2) on the surface $\xi=a$. Note that for the RL region one has $n^{l}=-\delta_{1}^{l}, K_{00}=-\xi$. To deal with discrete spectrum, we can introduce the second plate located at $\xi=b<a$, on whose surface we impose boundary conditions as well. After the construction of the corresponding partial zeta function we take the limit $b \rightarrow 0$. As a result we can see that the surface energymomentum tensor in the RL region has a structure given by (16) and with the equation of state (17). For the surface vacuum stress one obtains the expression

$$
p=\frac{B_{D}}{a^{D}} A(1-4 \zeta) I_{\mathrm{L}}(A), \quad A=-a A_{s},
$$

where now

$$
I_{\mathrm{L}}(A)=\left.F_{\mathrm{L}}(s)\right|_{s=0}, \quad F_{\mathrm{L}}(s)=\int_{0}^{\infty} d x x^{D-2} \zeta_{\mathrm{L}}(s, x)
$$

with

$$
\zeta_{\mathrm{L}}(s, x)=-\frac{1}{\pi} \cos \frac{\pi s}{2} \int_{\rho}^{\infty} d z z^{-s} \frac{I_{z}(x)}{\bar{I}_{z}(x)},
$$

and the barred notation is in accordance with Eq. (11). For a given $A$ this expression differs from the corresponding expression for the $\mathrm{RR}$ region by the replacement $K_{z}(x) \rightarrow I_{z}(x)$. Note that as in the previous section, to avoid the vacuum instability, here we have assumed $A_{s} \leq 0$. Under this condition, for a given $x$ the function $\bar{I}_{z}(x)$ has no real positive zeros with respect to $z$. By using the uniform asymptotic expansions for the Bessel modified function $I_{z}(x)$ and its derivative with respect to the argument, for the ratio in the subintegrand of expression (55) one receives

$$
\frac{I_{z}(x)}{\bar{I}_{z}(x)} \sim \frac{1}{\left(x^{2}+z^{2}\right)^{1 / 2}} \sum_{l=0}^{\infty} \frac{U_{l}(t)}{\left(1+x^{2}+z^{2}\right)^{l / 2}},
$$

with the same coefficients $U_{l}(t)$ as in Eq. (30). Now the expression for $F_{\mathrm{L}}(s)$ can be written as

$$
F_{\mathrm{L}}(s)=F_{\mathrm{L}}^{(a s)}(s)+F_{\mathrm{L}}^{(1)}(s)
$$

where

$$
\begin{aligned}
F_{\mathrm{L}}^{(a s)}(s) & =-\frac{1}{\pi} \cos \frac{\pi s}{2} \int_{0}^{\infty} d x x^{D-2} \int_{\rho}^{\infty} d z z^{-s} \frac{1}{r} \sum_{l=0}^{N} \frac{U_{l}(\cos \theta)}{\left(1+r^{2}\right)^{l / 2}} \\
F_{\mathrm{L}}^{(1)}(s) & =-\frac{1}{\pi} \cos \frac{\pi s}{2} \int_{0}^{\infty} d x x^{D-2} \int_{\rho}^{\infty} d z z^{-s}\left[\frac{I_{z}(x)}{\bar{I}_{z}(x)}-\frac{1}{r} \sum_{l=0}^{N} \frac{U_{l}(\cos \theta)}{\left(1+r^{2}\right)^{l / 2}}\right] .
\end{aligned}
$$

By the way similar to the case of the RR region, the asymptotic part can be presented in the form

$$
F_{\mathrm{L}}^{(a s)}(s)=-\frac{1}{\pi} \cos \frac{\pi s}{2} \sum_{l=1}^{N} B\left(\frac{D-s-1}{2}, \frac{l+s-D+1}{2}\right) \sum_{m=0}^{l} U_{l m} B\left(m-\frac{s-1}{2}, \frac{D-1}{2}\right) .
$$

The corresponding residue is easily found by using the formula for the gamma function:

$$
F_{\mathrm{L},-1}^{(a s)}=-\frac{2}{\pi} \Gamma\left(\frac{D-1}{2}\right) \sum_{j=0}^{j_{D}} \frac{(-1)^{j}}{\Gamma(j+1) \Gamma\left(\frac{D-1}{2}-j\right)} \sum_{m=0}^{D-2 j-1} U_{D-2 j-1, m} B\left(m+\frac{1}{2}, \frac{D-1}{2}\right),
$$


with $j_{D}$ defined by expression (43). Expanding near the simple pole $s=0$ we can write

$$
F_{\mathrm{L}}^{(a s)}(s)=\frac{F_{\mathrm{L},-1}^{(a s)}}{s}+F_{\mathrm{L}, 0}^{(a s)}+\mathcal{O}(s),
$$

with

$$
\begin{aligned}
F_{\mathrm{L}, 0}^{(a s)} & =-\frac{1}{\pi} \Gamma\left(\frac{D-1}{2}\right) \sum_{j=0}^{j_{D}} \frac{(-1)^{j}}{\Gamma(j+1) \Gamma\left(\frac{D-1}{2}-j\right)} \sum_{m=0}^{D-2 j-1} U_{D-2 j-1, m} B\left(m+\frac{1}{2}, \frac{D-1}{2}\right) \\
& \times\left[\psi\left(m+\frac{D}{2}\right)+\psi(j+1)-\psi\left(m+\frac{1}{2}\right)-\psi\left(\frac{D-1}{2}\right)\right] \\
& -\frac{1}{\pi}\left(\sum_{l=1, D-l=\text { even }}^{D-1}+\sum_{l=D}^{N}\right) B\left(\frac{l-D+1}{2}, \frac{D-1}{2}\right) \sum_{m=0}^{l} U_{l m} B\left(m+\frac{1}{2}, \frac{D-1}{2}\right),
\end{aligned}
$$

where the second sum in the braces of the third line is present only for $N \geq D$. Taking all contributions together, near $s=0$ we find

$$
F_{\mathrm{L}}(s)=\frac{F_{\mathrm{L},-1}^{(a s)}}{s}+F_{\mathrm{L}, 0}^{(a s)}+F_{\mathrm{L}}^{(1)}(0)+\mathcal{O}(s),
$$

with different terms defined by formulae (59), (61), (63). Note that for a given $A$ the pole parts of the surface densities for the RR and RL regions differ by the factor $(-1)^{D}$. Relation (64) allows us to present the vacuum stress as a sum of pole and finite parts:

$$
\begin{aligned}
p_{p}^{(\mathrm{L})} & =\frac{B_{D}}{s a^{D}} A(1-4 \zeta) F_{\mathrm{L},-1}^{(a s)}, \quad A=-a A_{s} \\
p_{f}^{(\mathrm{L})} & =\frac{B_{D}}{a^{D}} A(1-4 \zeta)\left[F_{\mathrm{L}, 0}^{(a s)}+F_{\mathrm{L}}^{(1)}(0)\right] .
\end{aligned}
$$

The corresponding decomposition of the surface energy density is obtained from here with the help of the equation of state (17). In Fig. 1, the finite part of $D=3$ surface vacuum stress $a^{D} p_{f}^{(\mathrm{L})} /[A(1-4 \zeta)]$ is presented for the RL region as a function of the parameter $-a A_{s}$. In the case of a minimally coupled scalar the corresponding surface energy-momentum tensor is a cosmological constant type located on the plate. As follows from Fig. 1, in $D=3$ the induced cosmological constant is negative for $0.378<A<1.941$ and is positive otherwise. For the Neumann boundary condition one has a relation similar to (50) with the replacement $\mathrm{R} \rightarrow \mathrm{L}$. In this case the finite part of the surface energy per unit area of the plate for $D=3$ is given by formula

$$
E_{\mathrm{N} f}^{(\mathrm{L}, \mathrm{surf})}=-\frac{0.142 \zeta}{a^{2}} .
$$

In accordance with Ref. [10] the corresponding part in the total vacuum energy is equal to $E_{\mathrm{N} f}^{(\mathrm{L})}=0.000792 / a^{2}$. For an infinitely thin plate taking the RR and RL regions together, the pole parts of the both surface and total vacuum energies cancel in $D=3$ and the corresponding Casimir energies are finite:

$$
E_{\mathrm{N}}^{(\text {surf })}=-\frac{0.0309 \zeta}{a^{2}}, \quad E_{\mathrm{N}}=\frac{0.00292}{a^{2}} .
$$

The relation of these quantities to the energies measured by a uniformly accelerated observer is discussed in Ref. [10]. 


\section{Conclusion}

In this paper we have investigated the surface Casimir densities generated by a single plate moving by uniform proper acceleration, assuming that the field is prepared in the Fulling-Rindler vacuum state. The corresponding volume vacuum expectation values of the energy-momentum tensor were investigated in Refs. [6,7] for the geometry of a single plate and in Ref. [8] in the case of two plates. Here we consider a scalar field with mixed boundary conditions and as a regularization method the zeta function technique is employed. In the case of a single plate geometry the right Rindler wedge is divided into two regions, referred as RR and RL regions. By using the Cauchy's theorem on residues, we have constructed an integral representations for the corresponding zeta functions in both these regions, which are well suited for the analytic continuation. Subtracting and adding to the integrands the leading terms of the corresponding uniform asymptotic expansions, we present the corresponding functions as a sum of two parts. The first one is convergent at the physical point and can be evaluated numerically. In the second, asymptotic part the pole contributions are given explicitly in terms of the beta function. As a consequence, the vacuum expectation values of the surface energy-momentum tensor for separate $\mathrm{RR}$ and $\mathrm{RL}$ regions contain pole and finite contributions. The remained pole term is a characteristic feature for the zeta function regularization method and has been found for many other cases of boundary geometries. For a minimally coupled scalar field, the surface energymomentum tensor induced by quantum vacuum effects corresponds to a source of a cosmological constant type located on the plate and with the cosmological constant determined by the proper acceleration of the plate. In the case of the Neumann boundary condition the finite parts of the surface vacuum stresses vanish for the both $\mathrm{RR}$ and $\mathrm{RL}$ regions. In $D=3$ spatial dimensions the corresponding surface energies are given by relations (52) and (67). In this case for an infinitely thin plate taking the RR and RL regions together the pole parts of the surface vacuum energies cancel and the total surface energy is finite. The corresponding total vacuum energy, including the surface and volume parts, is evaluated in Ref. [10]. By using the conformal relation between the Rindler and dS spacetimes and the results from [7], in Ref. [11] the vacuum expectation value of the bulk energy-momentum tensor is evaluated for a conformally coupled scalar field which satisfies the Robin boundary condition on a curved brane in dS spacetime. By making use the same technique and the conformal properties of the surface energy-momentum tensor, from the results of the present paper we can obtain the surface vacuum energy-momentum tensor

induced on the brane in $\mathrm{dS}$ spacetime, which is a conformal image of a uniformly accelerated plate in the Minkowski spacetime.

\section{Acknowledgement}

The work of A.A.S. was supported in part by the Armenian Ministry of Education and Science (Grant No. 0887).

\section{References}

[1] S. A. Fulling, Phys. Rev D 7, 2850 (1973); J. Phys. A 10, 917 (1977).

[2] W. G. Unruh, Phys. Rev. D 14, 870 (1976).

[3] D. G. Boulware, Phys. Rev. D 11, 1404 (1975).

[4] U. H. Gerlach, Phys. Rev. D 40, 1037 (1989); Phys. Rev. D 64, 105004 (2001); Found. Phys. 33, 179 (2003). 
[5] N. D. Birrell and P. C. W. Davies, Quantum Fields in Curved Space (Chambridge University Press, Cambridge, England, 1982).

[6] P. Candelas and D. Deutsch, Proc. R. Soc. Lond. A 354, 79 (1977).

[7] A. A. Saharian, Class. Quantum Grav. 19, 5039 (2002).

[8] R. M. Avagyan, A. A. Saharian, A. H. Yeranyan, Phys. Rev. D 66, 085023 (2002).

[9] A. A. Saharian, Izv. Akad. Nauk Arm. SSR. Mat. 22, 166 (1987) [Sov. J. Contemp. Math. Anal. 22, 70 (1987)]; A. A. Saharian, "The generalized Abel-Plana formula. Applications to Bessel functions and Casimir effect", Report No. IC/2000/14; hep-th/0002239.

[10] A. A. Saharian, R. S. Davtyan, and A. H. Yeranyan, Phys. Rev. D 69, (2004, in press), hep-th/0307163.

[11] A. A. Saharian and M. R. Setare, Phys. Lett. B584, 306 (2004).

[12] A. Romeo and A. A. Saharian, J. Phys. A 35, 1297 (2002).

[13] S. A. Fulling, J. Phys. A 36, 6857 (2003).

[14] A. A. Saharian, Phys. Rev. D 63, 125007 (2001).

[15] A. Romeo and A. A. Saharian, Phys. Rev. D 63, 105019 (2001).

[16] A. A. Saharian, Phys. Rev. D 69, (2004, in press), hep-th/0308108.

[17] J. S. Dowker and R. Critchley, Phys. Rev. D 13, 3224 (1976); S. W. Hawking, Commun. Math. Phys. 55, 133 (1977); G. W. Gibbons, Phys. Lett. B 60, 385 (1977).

[18] E. Elizalde, S. D. Odintsov, A. Romeo, A. A. Bytsenko, and S. Zerbini, Zeta Regularization Techniques with Applications (World Scientific, Singapore, 1994).

[19] V. Moretti, Phys. Rev. D 56, 7797 (1997); J. Math. Phys. 40, 3843 (1999).

[20] S. K. Blau, M. Visser, and A. Wipf, Nucl. Phys. B310, 1631 (1988).

[21] E. Elizalde, S. Leseduarte, and A. Romeo, J. Phys. A 26, 2409 (1993).

[22] S. Leseduarte and A. Romeo, J. Phys. A 27, 2483 (1994).

[23] M. Bordag, E. Elizalde, and K. Kirsten, J. Math. Phys. 37, 895 (1996).

[24] S. Leseduarte and A. Romeo, Ann. Phys. (N.Y.) 250, 448 (1996).

[25] M. Bordag, K. Kirsten, and J. S. Dowker, Commun. Math. Phys. 182, 371 (1996).

[26] M. Bordag, E. Elizalde, K. Kirsten, and S. Leseduarte, Phys. Rev. D 56, 4896 (1997).

[27] G. Lambiase, V. V. Nesterenko, and M. Bordag, J. Math. Phys. 40, 6254 (1999).

[28] G. Cognola, E. Elizalde, and K. Kirsten, J. Phys. A 34, 7311 (2001).

[29] M. Abramowitz and I. A. Stegun, Handbook of Mathematical functions (National Bureau of Standards, Washington D.C., 1964).

[30] J. C. Collins, Renormalization: An Introduction to Renormalization, the Renormalization Group, and the Operator Product Expansion (Cambridge University Press, Cambridge, 1984). 
[31] M. Bordag, V. V. Nesterenko, and I. G. Pirozhenko, Phys. Rev. D 65, 045011 (2002).

[32] V. V. Nesterenko, I. G. Pirozhenko, and I. Dittrich, Class. Quantum Grav. 20, 431 (2003). 